\title{
Energy Transfer in Macromolecular Arrays
}

\author{
David L. Andrews, Robert D. Jenkins \\ School of Chemical Sciences and Pharmacy, University of East Anglia, Norwich NR4 7TJ, UK
}

\begin{abstract}
Macromolecular systems comprised of many light-sensitive centres (the photosynthetic unit, dendrimers, and other highly symmetric multichromophore arrays) are important structures offering challenges to theoreticians and synthetic chemists alike. Here we outline novel photophysical interactions predicted and observed in such arrays. Using the tools of molecular quantum electrodynamics (QED) we present quantum amplitudes for a variety of higher-order resonance energy transfer (RET) schemes associated with well-known nonlinear optical effects such as two- and threephoton absorption. The initial analysis is extended to account for situations where the participant donor species are identical and exist in a highly symmetric environment, leading to the possible formation of excitons. It emerges from the QED theory that such excitons are closely associated with the higher-order RET processes. General results are interpreted by analyzing particular molecular architectures which offer interesting features such as rate enhancement or limitation and exciton pathway quenching. Applications in the areas of photosynthesis, molecular logic gates and lowintensity fluorescence energy transfer are predicted.
\end{abstract}

Keywords: energy transfer, exciton, bi-exciton, Förster, light-harvesting, photosynthesis, dendrimers, macromolecules

\section{INTRODUCTION}

In highly symmetric systems which comprise many similar chromophore centres the absorption of light can result not only in site-specific species excitation, but also in the formation of excitons. Many suitable environments - both natural and synthetic - exhibit these phenomena. Of these, the most well-known are the beautiful light harvesting centres in photosynthesising bacteria such as Rhodopseudomonas (Rps.) acidophila, Rhodospirillum molischianum and Rhodospirillum rubrum. ${ }^{1}$ For example, the peripheral light-harvesting complexes (LH2) in Rps. acidophila exhibit $C_{9}$ symmetry in both 800 and $850 \mathrm{~nm}$ absorbing bacteriochlorophyll sub-units. ${ }^{2}$ These structures collect photons and transfer them (via resonance energy transfer, RET) to the reaction centre (RC). Until recently, the RC was considered to be another highly symmetric superstructure; however recent investigations have shown it to be squashed into a slightly oval configuration, ${ }^{3}$ raising questions about the enforcement of physical symmetry arguments in accompanying theoretical studies.

Photosynthetic systems do not have a monopoly on fulfilling the pre-requisites for exciton formation; other organic and bioinorganic supermolecules are equally suitable. These synthesized structures offer the advantage of being controllable in both composition and structure. This high degree of synthetic management is advantageous as it offers a challenge for the molecular architect and surety for the theoretician. Excellent examples of these systems are offered by dendrimers and multichromophore arrays. It has long been known that dendrimers (highly symmetric, usually selfassembling molecular superstructures) provide an ideal environment for energy transfer studies; ${ }^{4,5}$ as the field has progressed the properties of the constituent chromophore sub-units and core have been chosen to facilitate studies of energy hopping behaviour (each hop being an RET event). A further group of compounds suitable for exciton formation are covalently linked, metalloporphyrin-based chromophore centres. ${ }^{6}$ These have been successfully designed to mimic behaviour observed in actual photosynthetic systems, ${ }^{7,8}$ and also as the basis for optical molecular switches.

In this work we highlight theoretical studies into the formation and subsequent transfer of both excitons and bi-excitons within the above systems. Using the tools of molecular quantum electrodynamics (QED), ${ }^{10}$ a recently developed statesequence technique $^{11}$ allows the correct formulation of the behaviour of both types of exciton transfer within an artificial three-fold symmetric edifice. New insight is offered into the behaviour of excitonic relaxation and a thorough investigation into the effect of molecular architecture is given. 


\section{EXCITON AND BI-EXCITON FORMATION}

Our investigation is based on a model light-harvesting system comprising three identical donor chromophores A, B and $\mathrm{C}$, potentially coupled to an acceptor species D which may or may not be chemically identical to the donors; a chemically distinct acceptor obviates problems of direct excitation along with the donors. Since the majority of dendrimers exhibit three-fold symmetry, and this is also a symmetry element present in the light-harvesting system of Rps. acidophila, it is an obvious structural element for a general model. As such we assume that each donor sits at the corner of an equilateral triangle with the acceptor in the middle (see figures 1 and 2). All system components are in the electronic ground state prior to photoexcitation.

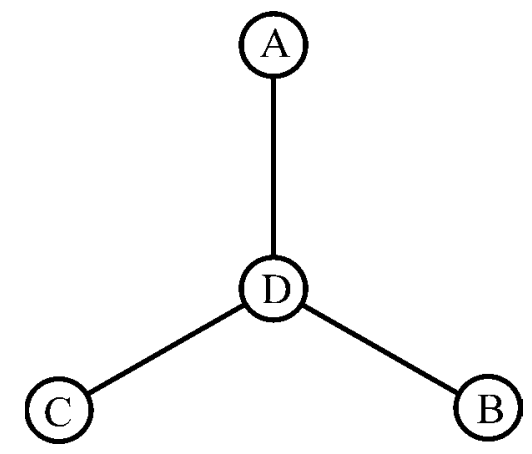

Figure 1: Three-fold symmetric model photosystem

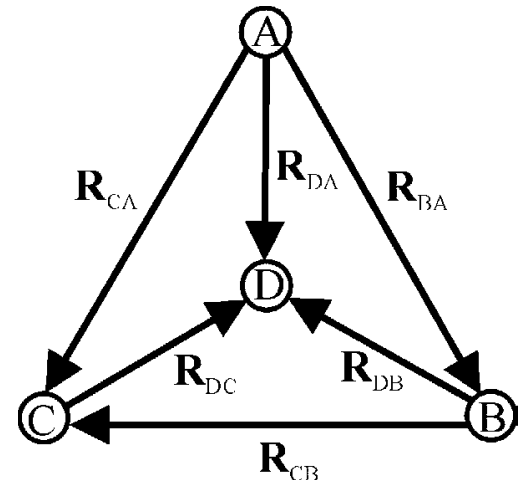

Figure 2: Inter-chromophore vectors in the model photosystem

\subsection{Single Photon Exciton}

When the model photosystem absorbs a photon of sufficient energy $h v$ to excite one of the donor species an exciton is formed, consistent with the system symmetry, signifying an uncertainty in localisation of the excitation. The energy of excitation is essentially accommodated in a state written as a superposition of chromophore molecular orbitals. It is this which constitutes the initial matter state for subsequent energy transfer, as compared to the localised excited donor state in conventional RET. We may express the photophysics through the non-chemical equation;

$$
\mathrm{A}^{0}+\mathrm{B}^{0}+\mathrm{C}^{0}+\mathrm{D}^{0}+h \nu \stackrel{\text { exciton formation }}{\longrightarrow}(\mathrm{A}+\mathrm{B}+\mathrm{C})^{*}+\mathrm{D}^{0} \stackrel{\text { energy trasfer }}{\longrightarrow} \mathrm{A}^{0}+\mathrm{B}^{0}+\mathrm{C}^{0}+\mathrm{D}^{u}
$$

where superscripts indicate the state in which a species resides $(0-$ ground state; $*$ - group excited state, $u$ - single species excited state). In (1) the exciton is represented by $(A+B+C)^{*}$ and the single asterisk indicates that one, undetermined donor is excited. Note that the process of exciton formation need not be immediate and can be associated with intramolecular relaxation following photoabsorption.

\subsection{Two-Photon Exciton (Bi-Exciton)}

Here two photons of approximately $h v$ are absorbed at different sites, forming the bi-exciton. The probability of achieving the necessary condition for bi-exciton formation is determined by the laser flux, and both the absorption cross-section $\sigma$ and the excited state lifetime of the donor, $\tau$. Generally, with $N$ donors and a laser delivering an irradiance $I$, the efficiency of bi-exciton formation is quantified by the ratio $N I \sigma \tau / h v$. With a very modest pulsed laser irradiance of $10^{12} \mathrm{~W} \mathrm{~cm}^{-2}$, an absorption cross-section of $10^{-18} \mathrm{~cm}^{2}$ and a lifetime of $100 \mathrm{fs}$, an bi-exciton formation efficiency of about $10 \%$ can be expected; obviously the exact figure is sensitive to the details of the system. Here the overall photophysics of bi-exciton formation and transfer is embodied in the equation;

$$
\mathrm{A}^{0}+\mathrm{B}^{0}+\mathrm{C}^{0}+\mathrm{D}^{0}+2 h v \stackrel{\text { exciton formation }}{\longrightarrow}(\mathrm{A}+\mathrm{B}+\mathrm{C})^{2^{*}}+\mathrm{D}^{0} \stackrel{\text { energy transfer }}{\longrightarrow} \mathrm{A}^{0}+\mathrm{B}^{0}+\mathrm{C}^{0}+\mathrm{D}^{u}
$$


In (2) the bi-exciton is represented by $(\mathrm{A}+\mathrm{B}+\mathrm{C})^{2^{*}}$; the superscript indicates that two, undetermined donors are excited. Again exciton formation need not be immediate.

In many photosystems, bi-excitonic energy transfer can compete directly with other possible relaxation processes. ${ }^{12}$ Those manifest at the exciton creation stage of (2) jeopardise the formation of $(A+B+C)^{2 *}$ by offering other absorption locations for the two input photons, electronic structure permitting. These alternatives could also serve as precursors to the desired acceptor excited state. For example, direct two-photon absorption (TPA) at the acceptor needs to be discounted. Furthermore, if the excitations were not absorbed by two donors but by one donor and the acceptor, singletsinglet (S-S) annihilation between the two chromophores would also result in the observed fluorescence; however, this option is precluded when acceptor electronic states are not amenable to single photon absorption of $h v$. This highlights the advantage of having donor and acceptor chromophores which are either chemically different, or are rendered electronically so by fields in their local environment. Finally, TPA at a donor followed by two-centre energy transfer to the acceptor might also account for the requisite energetics. This can be discounted by ensuring that donor chromophores exhibit no absorption band at approximately $2 h v$. Loss of excitation at the energy transfer stage is limited to S-S annihilation between two donors. Clearly, the acceptor would not experience the required excitation as the S-S annihilation precludes it. For the analysis in this paper we assume the satisfaction of these criteria which discount S-S annihilation and TPA effects. In systems where S-S annihilation and TPA are observed, bi-exciton energy transfer represents a competing process that must be accommodated into any rate analyses.

\section{EXCITONIC AND BI-EXCITONIC INITIAL STATES}

The total Hamiltonian $H$ for the exciton-forming donor species is;

$$
H=\sum_{\xi=\mathrm{A}, \mathrm{B}, \mathrm{C}} H_{\xi}+\sum_{\xi, \xi^{\prime}=\mathrm{A}, \mathrm{B}, \mathrm{C}} V_{\xi \xi^{\prime}}
$$

where $H_{\xi}$ is the Hamiltonian for donor $\xi$ and $V_{\xi \xi}$ is the interaction between $\xi$ and $\xi$ '. The explicit representation of the $V_{\xi \xi}$ coupling can, if required, be cast in quantum electrodynamical form, however such detail is necessary only in connection with the excitation transfer detailed below. The ground-state from which both the exciton and bi-exciton are elicited is represented as;

$$
\left|\psi_{\mathrm{A}}^{0}\right\rangle\left|\psi_{\mathrm{B}}^{0}\right\rangle\left|\psi_{\mathrm{C}}^{0}\right\rangle \equiv\left|\mathrm{A}^{0} \mathrm{~B}^{0} \mathrm{C}^{0}\right\rangle
$$

where $\psi_{\xi}^{0}$ is the ground-state wavefunction of $\xi$. The ground-state displacement energy $E_{0}$ is given by;

$$
E_{0}=\left\langle\mathrm{A}^{0} \mathrm{~B}^{0} \mathrm{C}^{0}|V| \mathrm{A}^{0} \mathrm{~B}^{0} \mathrm{C}^{0}\right\rangle,
$$

where $V$ is a short-hand for the second term in (3). In writing equation (5) we assume that the ground-state energy for any isolated donor species is zero; only interactions between the chromophores displace the absolute ground-state energy. The Heitler-London approximation delivers exciton eigenstates as linear combinations of donor eigenfunction products. Thus wavefunctions for $(\mathrm{A}+\mathrm{B}+\mathrm{C})^{*}$ and $(\mathrm{A}+\mathrm{B}+\mathrm{C})^{2^{*}}\left(\left|\Psi_{j}\right\rangle\right.$ and $\left.\left|\Psi^{\prime}{ }_{j}\right\rangle\right)$ can be written as the superpositions;

$$
\begin{gathered}
\left|\Psi_{j}\right\rangle=c_{j 1}\left|\mathrm{~A}^{u} \mathrm{~B}^{0} \mathrm{C}^{0}\right\rangle+c_{j 2}\left|\mathrm{~A}^{0} \mathrm{~B}^{u} \mathrm{C}^{0}\right\rangle+c_{j 3}\left|\mathrm{~A}^{0} \mathrm{~B}^{0} \mathrm{C}^{u}\right\rangle ; \\
\left|\Psi_{j}^{\prime}\right\rangle=c_{j 1}^{\prime}\left|\mathrm{A}^{u} \mathrm{~B}^{u} \mathrm{C}^{0}\right\rangle+c^{\prime}{ }_{j 2}\left|\mathrm{~A}^{u} \mathrm{~B}^{0} \mathrm{C}^{u}\right\rangle+c^{\prime}{ }_{j 3}\left|\mathrm{~A}^{0} \mathrm{~B}^{u} \mathrm{C}^{u}\right\rangle .
\end{gathered}
$$

In the above, the constants $c_{j n}$ and $c_{j n}^{\prime}$ are normalized and constitute an orthogonal set. The energy of the $j^{\text {th }}$ exciton and bi-exciton states, $E_{j}$, are given by the time-independent Schrödinger equation; assuming that species self-interactions are negligible, these are explicitly given in Table 1, which highlights a Davydov splitting of $3 v$ between the $j=1$ and 
$j=2$ and 3 states of either quasi-particle. ${ }^{13,14}$ The assumptions made above are valid for our idealized structure; in other cases, local variations in chromophore orientation and/or steric effects may shift state and transition energies (as for example in a protein environment).

\begin{tabular}{|l|l|l|}
\hline Energy & Exciton & Bi-exciton \\
\hline$E_{1}$ & $E+2 v$ & $2 E+2 v$ \\
\hline$E_{2}=E_{3}$ & $E-v$ & $2 E-v$ \\
\hline
\end{tabular}

Table 1: Exciton state energies, $\left\langle\xi^{u}\left|H_{\xi}\right| \xi^{u}\right\rangle=E$ and $\left\langle\xi^{u}|V| \xi^{\prime u}\right\rangle=v$.

Using the results in Table 1 we write the exciton and bi-exciton states in the general forms;

$$
\begin{aligned}
& \frac{1}{\sqrt{3}}\left(\left|\mathrm{~A}^{u} \mathrm{~B}^{0} \mathrm{C}^{0} \mathrm{D}^{0}\right\rangle+c^{ \pm}\left|\mathrm{A}^{0} \mathrm{~B}^{u} \mathrm{C}^{0} \mathrm{D}^{0}\right\rangle+c^{\mp}\left|\mathrm{A}^{0} \mathrm{~B}^{0} \mathrm{C}^{u} \mathrm{D}^{0}\right\rangle\right), \\
& \frac{1}{\sqrt{3}}\left(\left|\mathrm{~A}^{u} \mathrm{~B}^{u} \mathrm{C}^{0} \mathrm{D}^{0}\right\rangle+c^{ \pm}\left|\mathrm{A}^{u} \mathrm{~B}^{0} \mathrm{C}^{u} \mathrm{D}^{0}\right\rangle+c^{\mp}\left|\mathrm{A}^{0} \mathrm{~B}^{u} \mathrm{C}^{u} \mathrm{D}^{0}\right\rangle\right)
\end{aligned}
$$

We also introduce coefficients $c^{ \pm}$and $c^{\mp}$ as a means to generate each relevant state where ${ }^{15}$

$$
c^{ \pm}=1+c^{\prime}\left(-\frac{3}{2} \pm \mathrm{i} \frac{\sqrt{3}}{2}\right)
$$

Putting $c^{\prime}=0$ gives the $\left|\Psi_{1}\right\rangle$ and $\left|\Psi_{1}^{\prime}\right\rangle$ states; with $c^{\prime}=1$ the upper signs in (10) yield $\left|\Psi_{2}\right\rangle$ and $\left|\Psi_{2}^{\prime}\right\rangle$ and the lower, $\left|\Psi_{3}\right\rangle$ and $\left|\Psi^{\prime}{ }_{3}\right\rangle$. Note that the multiplicative coefficients form the characters of the table for representations of the cyclic group $C_{3}$, the rotational sub-group for the point group $D_{3 h}$ which describes the overall idealized dendrimer symmetry. For example the coefficients of $\left|\Psi_{1}\right\rangle$ are identical to the irreducible representation $A$ whilst those of $\left|\Psi_{2}\right\rangle$ and $\left|\Psi_{3}\right\rangle$ match the degenerate irreducible representations $E$.

\section{EXCITONIC ENERGY TRANSFER}

We now employ molecular QED to calculate the rate of energy transfer $\Gamma$ from the (single exciton) donor exciton states to the central acceptor. To begin, we address the simplest case, the exciton. The rate can be developed from a quantum amplitude $M_{f i}^{i ; j}$ which carries superscripts $i ; j$ to denote an $i$-fold symmetric system containing $j$ excitations. For transfer from an initial state $\left|r_{0}^{1}\right\rangle$, given by the linear combination shown in (8), the coupling of each of the three initial state component parts to the final state $\left|r_{2}^{1}\right\rangle$ must be accounted for. Any system basis state is a product of matter and radiation components, the matter part necessarily entailing a product of electronic states from all participant species. In the exciton case the quantum amplitude is given by the second-order time-dependent perturbation theory equation; ${ }^{16}$

$$
M_{f i}^{3 ; 1}=\sum_{m} \frac{\left\langle r_{2}^{1}\left|H_{\mathrm{int}}\right| r_{1}^{m}\right\rangle\left\langle r_{1}^{m}\left|H_{\mathrm{int}}\right| r_{0}^{1}\right\rangle}{\left(E_{r_{0}^{1}}-E_{r_{1}^{m}}\right)}
$$

where the total energy (both matter and radiation) of a state $|r\rangle$ is represented by $E_{r}$. In calculations that entail more that one radiation-matter interaction, energy non-conserving intermediate states are manifest as virtual molecular and/or radiation states. In (11) they take are represented by $\left|r_{k}^{m}\right\rangle$, where $m$ is a label to distinguish between them (see 
reference 11 for a full explanation of the significance of the $m$ values). Also present in (11) is the interaction Hamiltonian which is given by;

$$
H_{\mathrm{int}}=-\varepsilon_{0}^{-1} \mu(\xi) \cdot \mathbf{d}^{\perp}\left(\mathbf{R}_{\xi}\right)
$$

in the electric dipole approximation. In $(12), \mu(\xi)$ is the electric dipole moment operator for $\xi$ and $\mathbf{d}^{\perp}\left(\mathbf{R}_{\xi}\right)$ is the transverse electric field displacement operator evaluated at the position of $\xi, \mathbf{R}_{\xi}$. In order to account for the effects of any intervening medium a modification to the vacuum form of $\mathbf{d}^{\perp}\left(\mathbf{R}_{\xi}\right)$ must be used. Accordingly the energy transfer, which in vacuo is mediated by a virtual photon coupling, is now cast in terms of an exchange of virtual polaritons (medium-dressed virtual photons) which act as quanta of a dynamical sub-system comprising the radiation and normal modes of the host medium. Thus accounting for local field and other media corrections, the electric field displacement operator is given by; ${ }^{17}$

$$
\mathbf{d}^{\perp}\left(\mathbf{R}_{\xi}\right)=\mathrm{i} \sum_{\mathbf{q}} \sum_{s} \sum_{\lambda=1}^{2}\left(\frac{\varepsilon_{0} \hbar \omega_{q}^{(s)} v^{(s)}}{2 c V_{0} n^{(s)}}\right)^{\frac{1}{2}}\left(\frac{\left(n^{(s)}\right)^{2}+2}{3}\right)\left[\mathbf{e}^{(\lambda)}(\mathbf{q}) P_{\mathbf{q}, s, \lambda} \mathrm{e}^{\mathrm{iq} \cdot \mathbf{R}_{\xi}}-\overline{\mathbf{e}}^{(\lambda)}(\mathbf{q}) P_{\mathbf{q}, s, \lambda}^{\dagger} \mathrm{e}^{-\mathrm{iq} \cdot \mathbf{R}_{\xi}}\right]
$$

accommodating a summation over polariton modes, within a quantisation volume $V_{0}$, characterized by wave-vector $\mathbf{q}$, polarisation $\lambda$ and dispersion branch number $s$. Equation (3.5) also introduces the polariton polarisation unit vector $\mathbf{e}$ (and complex conjugate $\overline{\mathbf{e}}$ ), boson annihilation and creation operators $P$ and $P^{\dagger}$ respectively, and two quantities related to the polariton frequency $\omega_{q}^{(s)}$; the complex refractive index of the molecular medium $n^{\prime(s)}$ and the group velocity $v^{(s)}$. It is important to note that the media corrections concern the electronic influence of matter other than the chromophore set A, B, C, D and are therefore not the same as the interspecies interactions embodied in $v$.

The quantum amplitude is concisely expressible as follows (using the implied summation convention for repeated Cartesian indices);

$$
M_{f i}^{3 ; 1}=\frac{1}{\sqrt{3}}\left(\mu_{i}^{0 u(\mathrm{~A})} V_{i j}^{ \pm}\left(n K, \mathbf{R}_{\mathrm{DA}}\right) \mu_{j}^{\delta 0(\mathrm{D})}+c^{+} \mu_{i}^{0 u(\mathrm{~B})} V_{i j}^{ \pm}\left(n K, \mathbf{R}_{\mathrm{DB}}\right) \mu_{j}^{\delta 0(\mathrm{D})}+c^{-} \mu_{i}^{0 u(\mathrm{C})} V_{i j}^{ \pm}\left(n K, \mathbf{R}_{\mathrm{DC}}\right) \mu_{j}^{\delta 0(\mathrm{D})}\right)
$$

Here $\mu^{r^{\prime} r(\xi)}=\left\langle\xi^{r^{\prime}}|\mu(\xi)| \xi^{r}\right\rangle$ is the transition dipole of $\xi$ from state $|r\rangle$ to $\left|r^{\prime}\right\rangle$ and we introduce the interspecies vector displacements $\mathbf{R}_{\mathrm{D} \xi}=\mathbf{R}_{\mathrm{D}}-\mathbf{R}_{\xi}$. Also contained in (14) is a second-rank, index-symmetric tensor representing the media-corrected electric dipole-electric dipole coupling, explicitly;

$$
V_{i j}^{ \pm}(n K, \mathbf{R})=\frac{1}{n^{2}}\left(\frac{n^{2}+2}{3}\right) \frac{\mathrm{e}^{ \pm \mathrm{i} n \mathrm{~K} R}}{4 \pi \varepsilon_{0} R^{3}}\left\{(1 \mp \mathrm{i} n K R)\left(\delta_{i j}-3 \hat{R}_{i} \hat{R}_{j}\right)-(n K R)^{2}\left(\delta_{i j}-\hat{R}_{i} \hat{R}_{j}\right)\right\}
$$

describing the transfer of $h c K$ between the pre-excited donor and ground-state acceptor. The electronic influence of the intervening medium is evident in the Lorentz pre-multiplier and in the refractive index $n$ for the optical frequency $c K$. In real dispersive media $n$ carries an imaginary part, imposing an exponential decay through the phase factor. Necessarily, (15) reduces to the vacuum case as $n \rightarrow 0$. Also note the sign ambiguity in (15) which addresses problems associated with the outgoing wave approximation - a device widely used by previous authors to bring the quantum model into line. ${ }^{18}$ Here we are dealing with a quantum system and (15) correctly describes both incoming and outgoing waves - accommodating both time-orderings as a correct quantum description should. It is important not to lose sight of the fact that the interaction tensor is part of a quantum amplitude, a convenient construct but not a measurable. So long as the observable offers an accurate model of experimental data, then ambiguity at the quantum amplitude level is perfectly acceptable. 
Continuing, equation (14) exhibits three separate parts, one each for transfer from each donor involved in the exciton transfer to the acceptor. Previous work from this group has shown that these quantum relaxation routes are interlaced at the quantum level; ${ }^{16}$ the concise representation of equation (14) is only possible because it is cast in terms of the QED interaction tensor (15) that fully accommodates electronic effects of the intervening medium over all space.

\section{BI-EXCITONIC ENERGY TRANSFER}

A similar approach to that of the one-photon exciton discussed in section 4 can be adopted for the two-photon case, where two out of the three donors are excited in the initial state. The bi-exciton initial state is described by (9) and, due to the extra excitation present in each state, we use fourth-order time-dependent perturbation theory to describe the photophysics. Each contribution corresponds to a quantum amplitude for three-centre energy transfer (3CET).

Here three pathways exist — one so-called cooperative (coop) ${ }^{19}$ and two accretive (acc1 and acc2). ${ }^{20}$ Taking the first term in (9) as an example, we can see that A and B are the two excited species ( $\mathrm{C}$ is essentially dormant throughout this channel) - the three transfer pathways, in the context of the idealized dendrimer, are illustrated in figure 2.

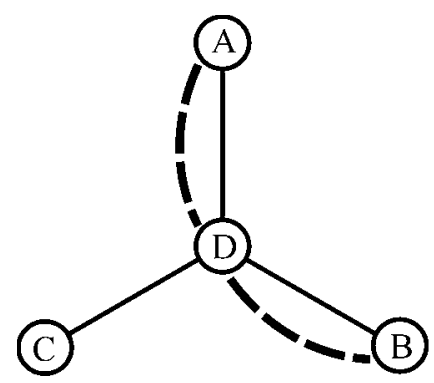

(a)

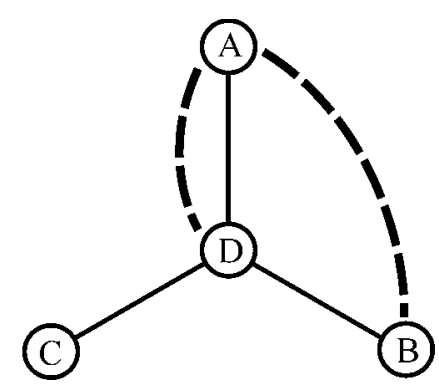

(b)

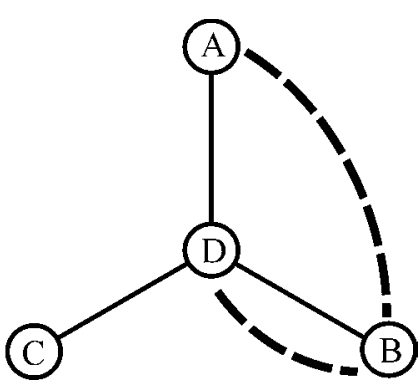

(c)

Figure 2: 3CET pathways contributing to $M_{f i}^{\mathrm{AB}}$ (a) coop, (b) acc1, (c) acc2. Here dotted lines join interaction-pairs. C is passive.

Adopting the language of interaction-pairs ${ }^{21}$ we can correctly describe the three 3CET pathways. Firstly the cooperative mechanism can be denoted by AD BD (figure 2a). The notation indicates that transfer occurs between the pairs $\mathrm{A}$ and $\mathrm{D}$ and between $\mathrm{B}$ and $\mathrm{D}$. It is a concerted interaction, i.e. the acceptor $\mathrm{D}$ has no energy level amenable to a single energy transfer from a single donor; only the combination of the two excitation transfers satisfies energy conservation. However D still undergoes a transition of two-photon character (vide infra), as is shown by the dual registration of D in the transfer-pairs AD BD. Similarly the accretive mechanisms are given by BA AD (figure $2 \mathrm{~b}$ ) and $\mathrm{AB} \mathrm{BD}$ (figure $2 \mathrm{c}$ ) respectively. In both these cases the total excitation in the system is fleetingly accrued by the donor species common in the two transfer pairs ( $\mathrm{A}$ in acc1 and $\mathrm{B}$ acc2) with the sum energy being deposited at the acceptor. Here it is the bridging donor (A or B) that undergoes the two-photon transition.

The total quantum amplitude for the dual excitation exciton transfer $M_{f i}^{3 ; 2}$ can be written as;

$$
M_{f i}^{3 ; 2}=M_{f i}^{\mathrm{AB}}+c^{ \pm} M_{f i}^{\mathrm{AC}}+c^{\mp} M_{f i}^{\mathrm{BC}}
$$

where each contributor exhibits a dormant donor species (one which remains unchanged, in the ground-state, throughout the interaction) and two excited participants $\xi$ and $\xi$ '. In general;

$$
M_{f i}^{\xi \xi^{\prime}}={ }^{\mathrm{coop}} M_{f i}^{\xi \xi^{\prime}}+{ }^{\mathrm{accl}} M_{f i}^{\xi \xi^{\prime}}+{ }^{\mathrm{acc} 2} M_{f i}^{\xi \xi^{\prime}}
$$


illustrating that a quantum amplitude comprises three parts, one for each transfer pathway, as in the examples given above. Using previously established techniques ${ }^{11,19}$ each quantum amplitude component in (17), and consequently the total $M_{f i}^{3 ; 2}$, may be calculated. Explicitly, the general form of the matrix element for each relaxation route is;

$$
\begin{gathered}
{ }^{\mathrm{coop}} M_{f i}^{\xi \xi^{\prime}}=\mu_{i}^{r^{\prime} r(\xi)} V_{i j}(n K, \mathbf{r}) \alpha_{j k}^{\delta 0(\mathrm{D})}(-c K,-c K) V_{k l}\left(n K, \mathbf{r}^{\prime}\right) \mu_{i}^{r^{r^{\prime}} r\left(\xi^{\prime}\right)}, \\
{ }^{\mathrm{acc1}} M_{f i}^{\xi \xi^{\prime}}=\mu_{i}^{r^{\prime} r(\xi)} V_{i j}\left(n K, \mathbf{r}^{\prime \prime}\right) \alpha_{j k}^{r^{\prime} r\left(\xi^{\prime}\right)}(2 c K,-c K) V_{k l}\left(2 n^{\prime} K, \mathbf{r}^{\prime}\right) \mu_{i}^{\delta 0(\mathrm{D})}, \\
{ }^{\mathrm{acc} 2} M_{f i}^{\xi^{\prime}}=\mu_{i}^{r^{\prime} r\left(\xi^{\prime}\right)} V_{i j}\left(n K, \mathbf{r}^{\prime \prime}\right) \alpha_{j k}^{r^{\prime} r(\xi)}(2 c K,-c K) V_{k l}\left(2 n^{\prime} K, \mathbf{r}\right) \mu_{i}^{\delta 0(\mathrm{D})},
\end{gathered}
$$

where $\mathbf{r}=\mathbf{r}_{\mathrm{D}}-\mathbf{r}_{\xi}, \mathbf{r}^{\prime}=\mathbf{r}_{\mathrm{D}}-\mathbf{r}_{\xi^{\prime}}, \mathbf{r}^{\prime \prime}=\mathbf{r}_{\xi^{\prime}}-\mathbf{r}_{\xi}$ and $n^{\prime}$ is the refractive index at the optical frequency $2 c K$. Also present here are two manifestations of the two-photon interaction tensor, given by;

$$
\alpha_{i j}^{r^{\prime} r(\xi)}\left(\mp c \kappa, \mp c \kappa^{\prime}\right)=\sum_{\zeta}\left\{\frac{\mu_{i}^{r^{\prime} \zeta(\xi)} \mu_{j}^{\zeta r(\xi)}}{\tilde{E}_{\xi}^{\zeta r} \pm \hbar c \kappa}+\frac{\mu_{j}^{r^{\prime} \zeta(\xi)} \mu_{i}^{\zeta r(\xi)}}{\tilde{E}_{\xi}^{\zeta r} \pm \hbar c \kappa^{\prime}}\right\},
$$

which also plays a controlling role in other photophysical processes such as two-photon absorption and Raman scattering. Equation (4.6) properly accommodates the finite lifetime of the virtual state of species $\xi$ (depicted by $\zeta$ ) through a phenomenological correction to the corresponding energy such that;

$$
\tilde{E}_{\xi}^{\zeta r}=E_{\xi}^{\zeta r}-\mathrm{i} \Gamma_{\zeta}
$$

where the state assumes a Lorentzian profile $\Gamma_{\zeta}$ representing its HWHM linewidth. The two-photon interaction tensor (22) plays different roles in the different mechanisms of 3CET; the cooperative pathways requires $\alpha$ to adopt the characteristics of a two-photon absorption process as the acceptor simultaneously "receives" two excitations (one from each participant donor). Conversely, in the accretive pathways, the behaviour of $\alpha$ is akin to the transition polarisability which mediates single-photon scattering processes. ${ }^{10}$ The donor species it relates to transiently acquires the excitation energy of a second photon during the process, the sum of both excitations being deposited at the acceptor.

\section{APPLICATION TO A PLANAR PHOTOSYSTEM}

\section{1: Single Photon Exciton}

We next adapt the above results to facilitate elucidation of the geometric dependence. Enforcing the necessary condition $n K R<<1$, signifying transfer-pair distances substantially less than the input optical wavelength, we reduce the transfer tensor, and hence the general results of (14), to their short-range form;

$$
M_{f i}^{3 ; 1}=\frac{1}{4 \sqrt{3} \pi \varepsilon_{0} n^{2} R^{3}}\left(\frac{n^{2}+1}{3}\right) \mu \mu\left\|\mu^{u 0(\mathrm{D})}\right\|\left(\kappa+c^{ \pm} \kappa^{\prime}+c^{\mp} \kappa^{\prime \prime}\right) .
$$

To most readily interpret the angle-dependence of the results in general form, in the magnitude of the transition moments for all three donor species are now assumed to be identical, $\left|\mu^{0 u(\mathrm{~A})}\right|=\left|\mu^{0 u(\mathrm{~B})}\right|=\left|\mu^{0 u(\mathrm{C})}\right|=|\mu|$, each centered on the apex of an equilateral triangle. Also the system architecture dictates that $R=R_{\mathrm{DA}}=R_{\mathrm{DB}}=R_{\mathrm{DC}}$. Introduced here are three variants on the well-known feature of conventional RET studies, the orientation factor kappa, ${ }^{22}$ here given by;

$$
\kappa_{\mathrm{AD}}=\cos \Theta_{\mathrm{AD}}-3 \cos \phi_{\mathrm{A}} \cos \phi_{\mathrm{D}}
$$




$$
\begin{aligned}
& \kappa_{\mathrm{BD}}=\cos \Theta_{\mathrm{BD}}-3 \cos \theta_{\mathrm{B}} \cos \theta_{\mathrm{D}}, \\
& \kappa_{\mathrm{CD}}=\cos \Theta_{\mathrm{CD}}-3 \cos \gamma_{\mathrm{C}} \cos \gamma_{\mathrm{D}} .
\end{aligned}
$$

The angles subtended by the various unit vectors involved are defined as;

$$
\begin{aligned}
\mu^{\xi} \cdot \mu^{\xi^{\prime}} & =\left|\mu^{\xi}\right|\left|\mu^{\xi^{\prime}}\right| \cos \Theta_{\xi^{\prime}} \\
\mu^{\xi} \cdot \mathbf{R}_{\mathrm{DA}} & =\left|\mu^{\xi}\right|\left|\mathbf{R}_{\mathrm{DA}}\right| \cos \phi_{\xi} \\
\mu^{\xi} \cdot \mathbf{R}_{\mathrm{DB}} & =\left|\mu^{\xi}\right|\left|\mathbf{R}_{\mathrm{DB}}\right| \cos \theta_{\xi} \\
\mu^{\xi} \cdot \mathbf{R}_{\mathrm{DC}} & =\left|\mu^{\xi}\right|\left|\mathbf{R}_{\mathrm{DC}}\right| \cos \gamma_{\xi}
\end{aligned}
$$

where $\mu^{\xi}$ is short-hand for the transition moment of the species $\xi$. Analysis of the above equations must accommodate the intrinsic symmetry of the donors. This is achieved by ensuring that the donor transition dipoles are mutually arranged as in figure 4 such that;

$$
\phi_{\mathrm{A}}=\theta_{\mathrm{B}}=\gamma_{\mathrm{C}} .
$$

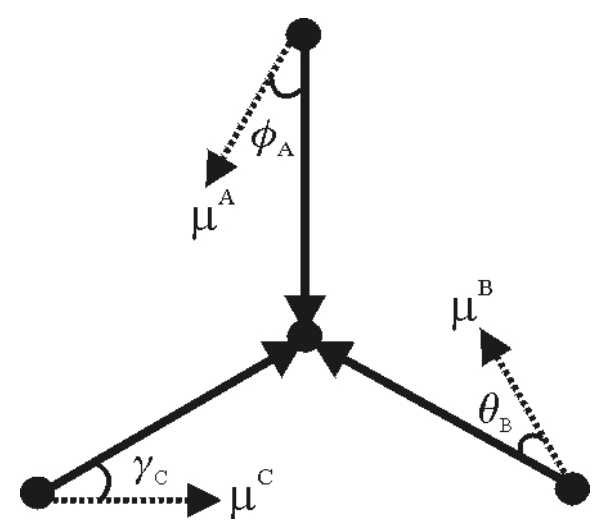

Figure 4: System geometry illustrating the case of $\phi_{\mathrm{A}}=\theta_{\mathrm{B}}=\gamma_{\mathrm{C}}$

Now we describe free, in-plane rotation of the acceptor transition moment with respect to model architecture in terms of the angle $\phi_{\mathrm{D}}$. Recognising that the criterion of (24) holds, recognize that;

$$
\phi_{\mathrm{D}}=\theta_{\mathrm{D}}-\frac{2 \pi}{3}=\gamma_{\mathrm{D}}-\frac{4 \pi}{3}
$$

Furthermore the angles between the donor and acceptor transition moments are expressible in terms of the angle $\Theta_{\mathrm{AD}}$;

$$
\Theta_{\mathrm{AD}}=\Theta_{\mathrm{BD}}-\frac{2 \pi}{3}=\Theta_{\mathrm{CD}}-\frac{4 \pi}{3}
$$

Using (24), (25) and (26) we may re-express (23) as;

$$
M_{f i}^{3 ; 1}=-\frac{\sqrt{3}\left|\mu \| \mu^{u 0(\mathrm{D})}\right|}{8 \pi \varepsilon_{0} R^{3}}\left\{\left(c^{ \pm}+c^{\mp}-2\right)\left(\cos \Theta_{\mathrm{AD}}-3 \cos \phi_{\mathrm{D}} \cos \phi_{\mathrm{A}}\right)+\sqrt{3}\left(c^{ \pm}-c^{\mp}\right)\left(\sin \Theta_{\mathrm{AD}}-3 \sin \phi_{\mathrm{D}} \cos \phi_{\mathrm{A}}\right)\right\} .
$$


In the excitonic state $\left|\Psi_{1}\right\rangle$ we have $c^{ \pm}=c^{\bar{\mp}}=1$, in which case equation (27) delivers a null result. Thus, energy transfer is forbidden from this excitonic state, no matter what the angular configurations in the planar dendrimer. For the degenerate states $\left|\Psi_{2}\right\rangle$ and $\left|\Psi_{3}\right\rangle$ the quantum amplitude takes two forms, one for each state, given by;

$$
M_{f i}^{3: 1( \pm)}=-\frac{\sqrt{3}|\mu|\left|\mu^{\mu 0(\mathrm{D})}\right|}{8 \pi \varepsilon_{0} R^{3}}\left\{\mathrm{e}^{ \pm \mathrm{I}_{\mathrm{AD}}}-3 \mathrm{e}^{ \pm \mathrm{i} \phi_{\mathrm{D}}} \cos \phi_{\mathrm{A}}\right\}
$$

where $M_{f i}^{3: 1(-)}$ is the quantum amplitude for $\left|\Psi_{2}\right\rangle$, and $M_{f i}^{3: 1(+)}$ that for $\left|\Psi_{3}\right\rangle$. The Fermi Golden Rule leads to the rate result;

$$
\Gamma=\frac{3|\mu|^{2}\left|\mu^{\mu 0(\mathrm{D})}\right|^{2} \rho_{f}}{32 \varepsilon_{0}^{2} \pi h R^{6}} f\left(\phi_{\mathrm{D}}, \phi_{\mathrm{A}}, \Theta_{\mathrm{AD}}\right)
$$

where $\rho_{f}$ is the density of acceptor final states and;

$$
f\left(\phi_{\mathrm{D}}, \phi_{\mathrm{A}}, \Theta_{\mathrm{AD}}\right)=\left|\mathrm{e}^{ \pm \mathrm{i} \Theta_{\mathrm{AD}}}-3 \mathrm{e}^{\mathrm{\pm i} \phi_{\mathrm{D}}} \cos \phi_{\mathrm{A}}\right|^{2}=\left(1-6 \cos \phi_{\mathrm{A}} \cos \left(\phi_{\mathrm{D}}-\Theta_{\mathrm{AD}}\right)+9 \cos ^{2} \phi_{\mathrm{A}}\right),
$$

proving that the observable for the degenerate excitons is independent of the \pm sign. It is revealing to produce threedimensional plots exhibiting the variation of $f\left(\phi_{\mathrm{D}}, \phi_{\mathrm{A}}, \Theta_{\mathrm{AD}}\right)$ with $\phi_{\mathrm{D}}$ and $\Theta_{\mathrm{AD}}$ at fixed $\phi_{\mathrm{A}} \cdot{ }^{15}$ It is obvious that when $\phi_{\mathrm{A}}=\pi / 2, f\left(\phi_{\mathrm{D}}, \phi_{\mathrm{A}}, \Theta_{\mathrm{AD}}\right)=1$ regardless of $\phi_{\mathrm{D}}$ and $\Theta_{\mathrm{AD}}$. Also, it is clear that the function in equation (30) is symmetrical about any $\phi_{\mathrm{D}}-\Theta_{\mathrm{AD}}=$ constant diagonal for any $\phi_{\mathrm{A}}$. As in conventional RET, orientations can be found to produce a zero rate, effectively "turning off" the transfer. The result of equation (24) is zero when;

$$
\cos \phi_{\mathrm{A}}=\frac{1}{3} \mathrm{e}^{\mathrm{i}\left(\phi_{\mathrm{D}}-\Theta_{\mathrm{AD}}\right)}
$$

which yields a real solution only when $\phi_{\mathrm{D}}=\Theta_{\mathrm{AD}}$. Thus, the configuration of $\phi_{\mathrm{A}}=\cos ^{-1}(1 / 3)$ and $\phi_{\mathrm{D}}=\Theta_{\mathrm{AD}}$ precludes energy transfer in the exciton system described above.

In fact this fully planar case affords further physical insight, as the principles it establishes can be extended to a system where the donor transition moments are no longer constrained to the system plane, given that threefold symmetry still applies amongst them. Here the donor moments comprise components both in and perpendicular to the plane. The above analysis shows that, for the totally symmetric donor exciton, the quantum amplitude for energy transfer to an inplane acceptor moment has a vanishing contribution from in-plane donor moment components. Furthermore contributions from perpendicular donor moment components must also vanish because they are orthogonal to both the acceptor dipole and each of the chromophore displacement vectors, as in the case of the out-of-plane acceptor moment. Consequently the principle of exclusion, concerning energy transfer from the totally symmetric exciton to an in-plane acceptor dipole, is generally valid and not limited to the case where all chromophore moments lie in the plane. ${ }^{15}$

\subsection{Bi-Exciton}

We now adapt the results for the bi-exciton, encapsulated in equation (16), to elicit geometric dependence. Here the conditions to reduce each transfer tensor to its short-range form are $2 n^{\prime} K R \approx n K R \quad<1$. Furthermore, for calculational simplicity, we assume both that all transition moments associated with individual species (either real or virtual) are collinear and $n \approx n^{\prime}$. Using techniques analogous to those in section 6.11 we can re-write the nine contributions to (16) in terms of angles rather that tensors/vectors; 


$$
\begin{aligned}
& { }^{\text {coop }} M_{f i}^{\xi^{\prime \prime}}=\frac{1}{n^{4}}\left(\frac{n^{2}+2}{3}\right)^{2} \frac{\left|\mu^{\xi}\left\|\mu^{\xi^{\prime}}\right\| \boldsymbol{\alpha}^{\mathrm{D}}\right| \kappa_{\xi \mathrm{D}} \kappa_{\xi^{\prime} \mathrm{D}}}{\left(4 \pi \varepsilon_{0} R^{3}\right)^{2}} \\
& { }^{\mathrm{accl} 1} M_{f i}^{\xi^{\prime}}=\frac{1}{n^{4}}\left(\frac{n^{2}+2}{3}\right)^{2} \frac{\left|\mu^{\xi}\left\|\mu^{\mathrm{D}}\right\| \boldsymbol{\alpha}^{\xi^{\prime}}\right| \boldsymbol{\kappa}_{\xi^{\prime}} \boldsymbol{\kappa}_{\xi^{\prime} \mathrm{D}}}{3^{\frac{3}{2}}\left(4 \pi \varepsilon_{0} R^{3}\right)^{2}} \\
& { }^{\mathrm{acc} 2} M_{f i}^{\xi^{\prime}}=\frac{1}{n^{4}}\left(\frac{n^{2}+2}{3}\right)^{2} \frac{\left|\boldsymbol{\mu}^{\xi^{\prime}}\left\|\boldsymbol{\mu}^{\mathrm{D}}\right\| \boldsymbol{\alpha}^{\xi}\right| \boldsymbol{\kappa}_{\xi \xi^{\prime}} \boldsymbol{\kappa}_{\xi \mathrm{D}}}{3^{\frac{3}{2}}\left(4 \pi \varepsilon_{0} R^{3}\right)^{2}}
\end{aligned}
$$

where we employ the intermolecular lengths of figure 2 and the short-hand notation $\mu^{f i(\xi)}=\mu^{\xi}$ and $\alpha^{f i(\xi)}=\alpha^{\xi}$. Obviously, due to the added complexity of the system, extra $\kappa$ factors are required to describe the behaviour. In addition to those in section 6.2 we define;

$$
\begin{aligned}
\kappa_{\mathrm{AB}} & =\cos \Theta_{\mathrm{AB}}-3 \cos \Lambda_{\mathrm{A}} \cos \Lambda_{\mathrm{B}} \\
\kappa_{\mathrm{AC}} & =\cos \Theta_{\mathrm{AC}}-3 \cos \Phi_{\mathrm{A}} \cos \Phi_{\mathrm{C}} \\
\kappa_{\mathrm{BC}} & =\cos \Theta_{\mathrm{BC}}-3 \cos \Xi_{\mathrm{B}} \cos \Xi_{\mathrm{C}}
\end{aligned}
$$

where;

$$
\begin{aligned}
\mu^{\xi} \cdot \mathbf{R}_{\mathrm{BA}} & =\left|\mu^{\xi}\right|\left|\mathbf{R}_{\mathrm{BA}}\right| \cos \Lambda_{\xi} \\
\mu^{\xi} \cdot \mathbf{R}_{\mathrm{CA}} & =\left|\mu^{\xi}\right|\left|\mathbf{R}_{\mathrm{CA}}\right| \cos \Phi_{\xi} \\
\mu^{\xi} \cdot \mathbf{R}_{\mathrm{CB}} & =\left|\mu^{\xi}\right| \mathbf{R}_{\mathrm{CB}} \mid \cos \Xi_{\xi} .
\end{aligned}
$$

Analysis of the above equations must again accommodate the intrinsic symmetry of the equilateral triangle formed by the donors - ensuring that equation (24) holds. In the co-planar architecture we may describe the system in terms of only three angles. We portray free, in-plane rotation of the acceptor transition moments with respect to the donoracceptor intermolecular vectors in terms of the angle $\phi_{\mathrm{D}}$ and the angles between the donor and acceptor transition moments can be expressed in terms of the angle $\Theta_{\mathrm{AD}}$ alone.

$$
\begin{gathered}
\phi_{\mathrm{D}}=\theta_{\mathrm{D}}-\frac{2 \pi}{3}=\gamma_{\mathrm{D}}-\frac{4 \pi}{3}, \\
\Theta_{\mathrm{AD}}=\Theta_{\mathrm{BD}}-\frac{4 \pi}{3}=\Theta_{\mathrm{CD}}-\frac{2 \pi}{3} .
\end{gathered}
$$

Finally we define all other relevant angles (those between donor transition moments and donor-donor intermolecular vectors) in terms of $\phi_{\mathrm{A}}$;

$$
\phi_{\mathrm{A}}=\Lambda_{\mathrm{A}}-\frac{\pi}{6}=\Lambda_{\mathrm{B}}+\frac{7 \pi}{6}=\Phi_{\mathrm{A}}+\frac{\pi}{6}=\Phi_{\mathrm{C}}+\frac{5 \pi}{6}=\Xi_{\mathrm{B}}-\frac{\pi}{6}=\Xi_{\mathrm{C}}+\frac{7 \pi}{6} .
$$

Noting that, due to the planarity of the system;

$$
\begin{gathered}
\kappa_{\mathrm{AD}}+\kappa_{\mathrm{BD}}=-\kappa_{\mathrm{CD}} \\
\kappa_{\mathrm{AB}}=\kappa_{\mathrm{AC}}=\kappa_{\mathrm{BC}}=3 \cos ^{2} \phi_{\mathrm{A}}-\frac{5}{4}
\end{gathered}
$$

we may write the total quantum amplitude as; 


$$
M_{f i}^{3 ; 2}=\frac{\left|\mu^{\mathrm{A}}\right|^{2}\left|\alpha^{\mathrm{D}}\right|}{\left(4 \pi \varepsilon_{0} R^{3}\right)^{2}}\left\{\kappa_{\mathrm{CD}} \kappa_{\mathrm{AD}}\left(c^{ \pm}-c^{\mp}-1\right)-c^{\mp} \kappa_{\mathrm{CD}}^{2}-\kappa_{\mathrm{AD}}^{2}\right\}+\frac{\left|\mu^{\mathrm{A}}\left\|\mu^{\mathrm{D}}\right\| \alpha^{\mathrm{A}}\right| \kappa_{\mathrm{AB}}}{3^{\frac{3}{2}}\left(4 \pi \varepsilon_{0} R^{3}\right)^{2}}\left\{\kappa_{\mathrm{CD}}\left(c^{ \pm}-1\right)+\kappa_{\mathrm{AD}}\left(c^{ \pm}-c^{\mp}\right)\right\}
$$

where the first term exclusively addresses the cooperative contributions, the second describes the accretive. Again, in the excitonic state $\left|\Psi_{1}\right\rangle$ we observe that $c^{ \pm}=c^{\mp}=1$, and the accretive part of equation (36) vanishes. This implies that biexcitronic transfer from the symmetrical state only proceeds via coop-based mechanisms. Substituting in the explicit relationships for the kappa factors, (36) becomes;

$$
M_{f i}^{3: 2}\left(\Psi_{1}\right)=-\frac{3\left|\mu^{\mathrm{A}}\right|^{2}\left|\alpha^{\mathrm{D}}\right|}{\left(8 \pi \varepsilon_{0} R^{3}\right)^{2}} f\left(\phi_{\mathrm{D}}, \phi_{\mathrm{A}}, \Theta_{\mathrm{AD}}\right)
$$

This result mirrors rate results for the degenerate states of the single-photon exciton states.

Calculation of the rate expressions using the Fermi Golden rule entails modulus squaring the sum of the relevant quantum amplitude contributions. As the results for the single photon exciton contain only a single $\kappa$ factor in the relevant quantum amplitudes, the corresponding rate expressions go as $\kappa^{2}$, the behaviour seen in the quantum amplitude of (23). Even though the number of angles needed to describe the bi-excitonic system is greater than those required for the single-photon exciton, the system symmetry dictates a directly comparable geometric dependence. However in single-photon excitons, energy transfer from the totally symmetric exciton to an in-plane acceptor dipole is forbidden. This selection rule is broken for the bi-exciton. Interestingly, transfer emanates from the cooperative mechanism, where symmetry is preserved, and not from the symmetry-breaking accretive pathways.

For the degenerate bi-exciton states $\left|\Psi_{2}\right\rangle$ and $\left|\Psi_{3}\right\rangle$, the quantum amplitude of (36) takes the form;

$$
M_{f i}^{3 ; 2}\left(\Psi^{ \pm}\right)=\frac{\left|\mu^{\mathrm{A}}\right|^{2}\left|\alpha^{\mathrm{D}}\right|}{\left(4 \pi \varepsilon_{0} R^{3}\right)^{2}}\left\{\kappa_{\mathrm{CD}}^{2}\left(\frac{1}{2} \pm \mathrm{i} \frac{\sqrt{3}}{2}\right)-\kappa_{\mathrm{AD}}^{2}+\kappa_{\mathrm{CD}} \kappa_{\mathrm{AD}}(-1 \pm \mathrm{i} \sqrt{3})\right\}+\frac{\left|\mu^{\mathrm{A}}\left\|\mu^{\mathrm{D}}\right\| \alpha^{\mathrm{A}}\right| \kappa_{\mathrm{AB}}}{2 \sqrt{3}\left(4 \pi \varepsilon_{0} R^{3}\right)^{2}}\left[\left(-\frac{3}{2} \pm \mathrm{i} \frac{\sqrt{3}}{2}\right) \kappa_{\mathrm{CD}} \pm \mathrm{i} \sqrt{3} \kappa_{\mathrm{AD}}\right]
$$

Here $M_{f i}^{3 ; 2}\left(\Psi^{+}\right)$and $M_{f i}^{3 ; 2}\left(\Psi^{-}\right)$represent the quantum pathways for energy transfer from $\left|\Psi_{2}\right\rangle$ and $\left|\Psi_{3}\right\rangle$ respectively. In (26) the first term again reports information on cooperative mechanisms and the second the accretive. From (38) and (27) we have;

$$
\begin{gathered}
\Gamma^{3 ; 2}\left(\Psi^{ \pm}\right)=\frac{\left|\mu^{\mathrm{A}}\right|^{4}\left|\alpha^{\mathrm{D}}\right|^{2}}{\left(4 \pi \varepsilon_{0} R^{3}\right)^{4}}\left\{\kappa_{\mathrm{AD}}^{4}+\kappa_{\mathrm{AD}}^{3} \kappa_{\mathrm{CD}}+\kappa_{\mathrm{AD}}^{2} \kappa_{\mathrm{CD}}^{2}+\kappa_{\mathrm{AD}} \kappa_{\mathrm{CD}}^{2}+\kappa_{\mathrm{CD}}^{4}\right\}+\frac{\sqrt{3}\left|\mu^{\mathrm{A}}\right|^{3}\left|\mu^{\mathrm{D}}\left\|\alpha^{\mathrm{A}}\right\| \alpha^{\mathrm{D}}\right| \kappa_{\mathrm{AB}}}{2\left(4 \pi \varepsilon_{0} R^{3}\right)^{2}}\left(3 \kappa_{\mathrm{AD}}^{2} \kappa_{\mathrm{CD}}+5 \kappa_{\mathrm{AD}} \kappa_{\mathrm{CD}}^{2}+\frac{3}{2} \kappa_{\mathrm{CD}}^{2}\right) \\
+\frac{\left|\mu^{\mathrm{A}}\right|^{2}\left|\mu^{\mathrm{D}}\right|^{2}\left|\alpha^{\mathrm{A}}\right|^{2} \kappa_{\mathrm{AB}}^{2}}{4\left(4 \pi \varepsilon_{0} R^{3}\right)^{2}}\left(\frac{7}{4} \kappa_{\mathrm{CD}}^{2}+\kappa_{\mathrm{AD}}^{2}+2 \kappa_{\mathrm{AD}} \kappa_{\mathrm{CD}}\right)
\end{gathered}
$$

where the first and third terms represent pure cooperative and accretive contributions respectively, and the second term quantifies their quantum interference. Concentrating on the angular dependences in equation (28), we may ascertain values that equate to optimum transfer probabilities for each contribution. We observe that it is the cooperative contribution that has the greatest influence, with the cross-term offering a small (but not insubstantial) input, with the accretive contribution being effectively negligible. This is to be expected, as a donor separation angle of $2 \pi / 3$ gives a significant bias toward cooperative transfer. More detailed results are to be presented elsewhere. ${ }^{23}$

\section{ACKNOWLEDGEMENTS}

This work was funded by the Engineering and Physical Sciences Research Council. 


\section{REFERENCES}

1. H. van Amerongen, L. Valkunas and R. van Grondelle, Photosynthetic excitons, World Scientific, Singapore, 2000.

2. G. McDermott, S. M. Prince, A. A. Freer, A. M. Hawthornthwaite-Lawless, M. Z. Papiz, R. J. Cogdell and N. W. Isaacs, Crystal structure of an integral membrane light-harvesting complex from photosynthetic bacteria, Nature, 374, 517, 1995.

3. S. Scheuring, J. Seguin, S. Marco, D. Lévy, B. Robert and J.-L. Rigaud, Nanodissection and high-resolution imaging of the Rhodopseudomonas viridis photosynthetic core complex in native membranes by AFM, PNAS, 100, 1690, 2003.

4. S. F. Swallen, Z.-Y. Shi, W. Tan, Z. Xu, J. S. Moore and R. Kopelman, Exciton localisation hierarchy and directed energy transfer in conjugated linear aromatic chains and dendrimeric supermolecules, J. Lumin., 76\&77, $193,1998$.

5. M. Maus, R. De, M. Lor, T. Weil, S. Mitra, U.-M. Wiesler, A. Herrmann, J. Vosch, K. Müllen and F. C. De Scryver, Intramolecular energy hopping and energy trapping in polyphenylene dendrimers with multiple peryleneimide donor chromophores and a terryleneimide acceptor trap chromophores, J. Am. Chem. Soc., 123, 7668, 2001.

6. P. Brodard, S. Matzinger, E. Vauthey, O. Mongin, C. Papamichaël and A. Gassauer, Investigations of electronic energy transfer dynamics in multiporphyrin arrays, J. Phys. Chem A, 103, 5858, 1999.

7. P. G. Van Patten, A. P. Shreve, J. S. Lindsey and R. J. Donohoe, Energy-transfer modelling for the rational design of multiporphyrin light-harvesting arrays, J. Phys. Chem. B, 102, 4209, 1998.

8. D. Kuciauskas, P. A. Liddell, S. Lin, T. E. Johnson, S. J. Weghorn, J. S. Lindsey, A. L. Moore, T. A. Moore and D. Gust, An artificial photosynthetic antenna-reaction center complex, J. Am. Chem. Soc., 121, 8604, 1999.

9. R. K. Lammi, R. W. Wagner, A. Ambroise, J. R. Diers, D. F. Bocian, D. Holten and J. S. Lindsey, Mechanisms of excited-state energy-transfer gating in linear versus branched multiporphyrin arrays, J. Phys. Chem. B, 105, 5341, 2001.

10. D. P. Craig and T. Thirunamachandran, Molecular Quantum Electrodynamics: An Introduction to Radiation Molecule Interactions, Dover, Mineola, 1998.

11. R. D. Jenkins, D. L. Andrews and L. C. Dávila Romero, A new diagrammatic methodology for non-relativistic quantum electrodynamics, J. Phys. B: At. Mol. Opt. Phys., 35, 445, 2002.

12. L. Valkunas, G. Trinkunas, V. Liuolia and R. van Grondelle, Nonlinear annihilation of excitations in photosynthetic systems. Biophys. J., 69, 1117, 1995.

13. V. Ćápek, I. Barvík and P. Heřman, Towards proper parametrisation in the exciton transfer and relaxation problem: Dimer, Chem. Phys., 270, 141, 2001.

14. P. Heřman and I. Barvík, Towards proper parametrisation in the exciton transfer and relaxation problem. II. Trimer, Chem. Phys., 274, 199, 2001.

15. R. D. Jenkins and D. L. Andrews, Exciton Resonance Energy Transfer: Effects of Geometry and Transition Moment Orientation in Model Photosystems, Photochem. Photobiol. Sci., 2, 103, 2003.

16. R. D. Jenkins and D. L. Andrews, Multichromophore excitons and resonance energy transfer: Molecular quantum electrodynamics, J. Chem. Phys., 118, 3470, 2003.

17. G. Juzeliūnas and D. L. Andrews, Quantum electrodynamics of resonance energy transfer, Adv. Chem. Phys., 112, $357,2000$.

18. G. J. Daniels, R. D. Jenkins, D. S. Bradshaw and D. L. Andrews, Resonance energy transfer: The Unified Theory revisited, J. Chem. Phys., 119, 2264, 2003.

19. R. D. Jenkins and D. L. Andrews, Three-center systems for energy pooling: Quantum electrodynamical theory, $J$. Phys. Chem. A, 102, 10834, 1998.

20. R. D. Jenkins and D. L. Andrews, Twin-donor systems for resonance energy transfer, Chem. Phys. Letters, 301, 235, 1999.

21. R. D. Jenkins and D. L. Andrews, Four-centre energy transfer and interaction pairs: Molecular quantum electrodynamics, J. Chem. Phys., 116, 6713, 2002.

22. B. W. Van der Meer, in Resonance Energy Transfer, eds. D. L. Andrews and A. A. Demidov, John Wiley and Son, Chichester, 1999.

23. R. D. Jenkins and D. L. Andrews, Bi-exciton resonance energy transfer: Effects of geometry and transition moment orientation in model photosystems, submitted for publication, (2003). 